\title{
Environmental Condition and Its Impact on Landscape Description by Salient Element
}

\author{
S. Soleimani ${ }^{\text {a* }}$, M.R. Malek ${ }^{\mathrm{a}}$, Z. Soleimani $^{\mathrm{b}}$, R. Arabsheibani ${ }^{\mathrm{c}}$ \\ ${ }^{a}$ Faculty of Geodesy and Geomatics Engineering, K.N. Toosi University of Technology, Tehran, Iran \\ ssoleimani@mail.kntu.ac.ir,m.malek@kntu.ac.ir \\ ${ }^{\mathrm{b}}$ Civil Department, University of Ayatollah Ozma Borujerdi, Borujerd, Iran \\ soleimani.zahra73@gmail.com \\ ${ }^{c}$ Department of Surveying and Geomatics Engineering, College of Engineering, University of Tehran \\ rasheibani@ut.ac.ir
}

KEYWORDS: Volunteered Geographical Information, Environmental condition, Data credibility, Landscape description, Salient element

\begin{abstract}
:
Describing a landscape means making link between concepts of visible features and people's perception. Most landscape description methods underline salient entities which are a key trigger for wayfinding problems and tourism management. Searching for a better understanding of landscape descriptions implies to explore and identify the main visual properties that differentiate between landscapes depending on both human cognition and environmental condition. Furthermore, this environmental condition affects the credibility of data produced by people, particularly when using Volunteered Geographical Information systems which brings forward a huge amount of information. Then this paper proposes an approach to emerge patterns by which describing landscape in general and choosing salient objects in particular have been influenced.
\end{abstract}

\section{INTRODUCTION}

For decades there is still a raised question to find the way how visual selection is performed in various landscapes by different individuals. However landscape definition mostly refers to the visible features of an area of land to be considered as recreational areas, the notion of landscape is illuminated by the concepts of land and nature, landmarks and people's perception (Litton 1968; Derungs, 2013). Then it is very difficult to describe it because of the role of people's cast of mind and sense of taste that is under influence of personal experience. Searching for a better understanding of landscape descriptions implies to explore and identify the main visual properties that differentiate between landscapes. Despite spatial configuration (Schirpke et al, 2013), spatial composition, and perceptive situation of observers that are needed to describe surrounded environment, it still remains absent the information about environmental condition to obtain how landscapes could be described.

Environmental condition is under such conditions of, not limited to, weather, temperature \& humidity, lighting, noise, odour, elevation, and even landscape each of which affects the perception of environment following by various implied quality. While main issue in acquiring the landscape descriptions is that perception of land varies from person to person, from time to time, and from condition to condition depending on the way people think or experience their surroundings.

Indeed, verbal description of a landscape generally underlines salient entities (Yaouanc et al., 2010). However, more salient and unexplained phenomena of modern psychophysics is the streaky appearance of human, but we can mention colour, area and distance as a key to distinguish salient component. In fact, salient elements help managers to make better decision when need exists, such as tourism management, wayfinding, and crisis management, to name but three.
According to the previous study, since visual saliency has a significant role in natural movement (Zhang and Liu, 2014), many computational models have been built to product saliency and cognitive maps. Zhang and Liu (2014) devised a computational model of visual attention in which the similarity between the phenomena and regions had been matched to find weather a specific phenomenon can be considered as a salience one or not. In another research, considering the fact that visual selection can be triggered by a salience-driven or a goal-driven account, Siebold and Mieke (2014) have investigated the role of timing of a response in order to address the problem of what guides visual selection. In goal-driven procedure the eye movements in real-world scenes are affected by information content rather than salience phenomena; however, eye movements are additionally salience driven, especially in the presence of phenomena that are salient enough to attract attention and the eyes (Siebold and Mieke, 2014). The main objective of a salient-driven process is to rapidly and accurately detect a phenomenon as more attractive one in a landscape (Yildirim and Sabine, 2014). Moreover, as for landform categories, Williams at al. (2015) have mentioned in their study that according to the observation of participant landform descriptions, the influence of salient perceptual features of the landscape, and landscape affordance or utilitarian motivations specifies landform categories.

The aim is to emerge the way environmental conditions play role in choosing salient element in a landscape that is more challenging when using crowdsourcing process. Therefore, there is too much data thanks to diversity of conditions observer faces during describing a landscape; however, the concern of reliability of gathered data is still raised (Flanagin and Metzger, 2008).

Actually, when it comes to reliability and credibility of crowedsourced data, knowing the human-environment relations is the first step to create a participatory environment by which individuals would easily air their view about different landscape

\footnotetext{
* Corresponding author
} 
and share it with each other (Forati et al., 2015). This condition improves the opportunity of having more reliable data to use them in further research.

\section{ENVIRONMENTAL CONDITIONS AFFECT LANDSCAPE DESCRIPTION}

There are many natural and artificial phenomena that can affect the human's cognition and therefore influence one's landscape descriptions (Cohen, 2013). Comprehensive assessment of environmental condition is a great challenge according upon various aspects of environment such as soil, biodiversity, water conservation, noise, and landscape aesthetics (Singer, 2014). These mentioned environmental variables or aspects play a significant role in environmental conditions. Their impact are easily noticeable, however they are not easily measured (Singer, 2014). Three of the main environmental conditions that can affect one's cognition are weather, light and type of landscape (Figure 1).

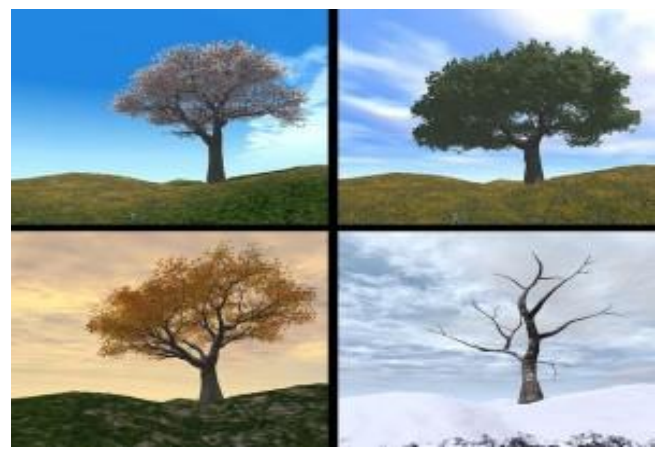

Weather

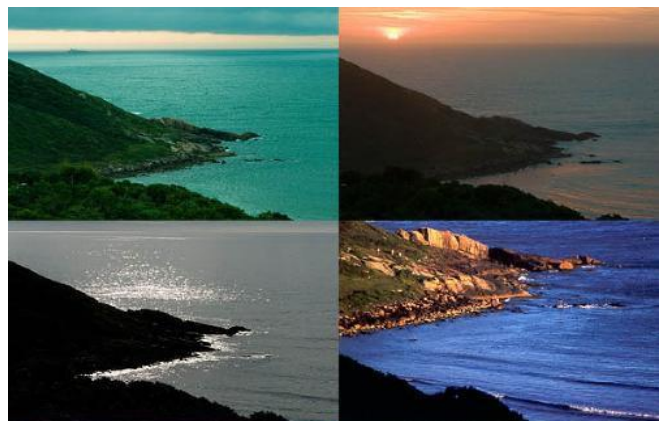

Light

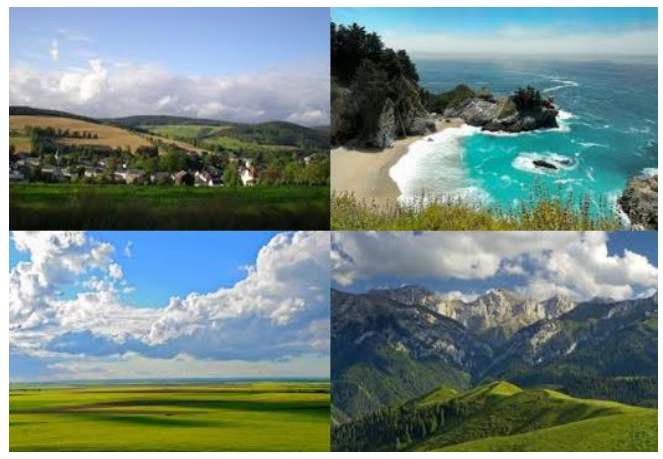

Landscape types

Figure 1. Some of different environmental condition when describing a landscape
Weather: needless to say that various types of weathers have a significant impact on how a landscape visually seems. There are different types of landscape including rainy, windy, sunny, lightening, cloudy, and snowy that each of which affects the way a specific landscape appears. We can see that how snow and snowy weathers changes specific landscape in the winter. Take a mountainous landscape as an example, there is a huge amount of difference between rainy weather views and snowy weather views; the snowy weather accentuates the mountains while they are de-emphasised when in rainy weather. In another word, weather is one of factors which guide visual and salience object selections.

Light: the time when a landscape is about to be described is a very important factor by which the whole of description might be completely changed. Suffice to say that light is the first and more important factor that makes a landscape visible for us. Furthermore, a specific landscape would be visually seen differently in various part of a day. For example, the visual selection revolves around shiny objects during a night, while the salience objects of that same landscape would be kind of colourful object during a day. This situation changes descriptions of a landscape.

Type of landscape: A catholic collection of landscape types has its effects of the way visual selection goes and salient objects are chosen. From among different types of landscape, mountain, forest, sea, field, and town are of the most abundant ones. Apart from the cognition process of human's mind and his/her background and interests, when facing with a specific type of landscape individual tries to select an object as a salience one that has a noticeable difference with that scene.

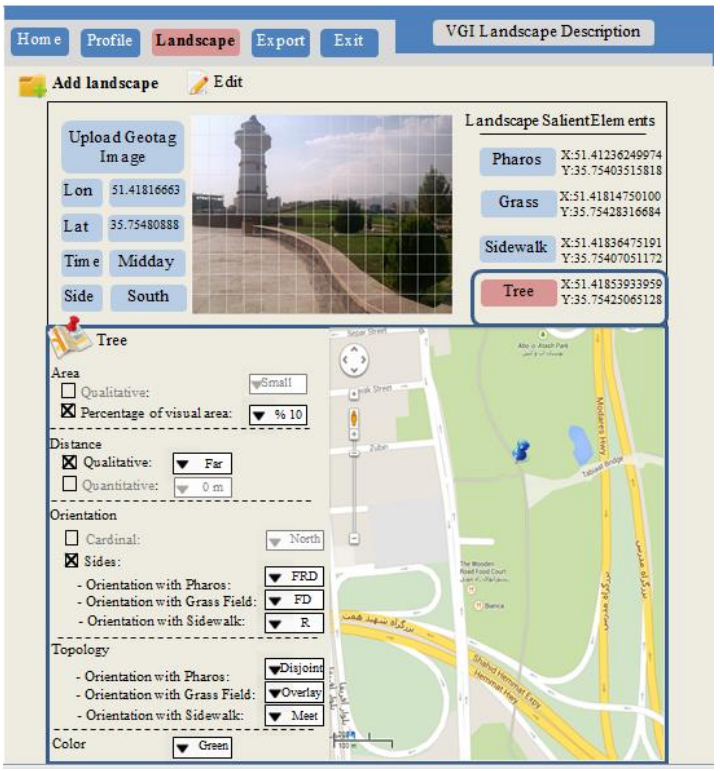

Figure 2. The web-based platform designed to collect the landscape descriptions

\section{PROPOSED APPROACH}

Generally, in order to address how the one's surrounding description following by his/her cognition affects the salient object selection process, coming forth with a huge data is concerned. To get along with this concern, a VGI based framework is designed with some experimented metrics needed to describe visual aspect of a landscape (Soleimani et al, 2015). 
In our suggested methodology some steps have been taken as follows (Figure 3): in order to evaluate the respective importance of the different metrics identified, an interactive VGI platform and interface has been designed and developed (Figure 2). The designed VGI

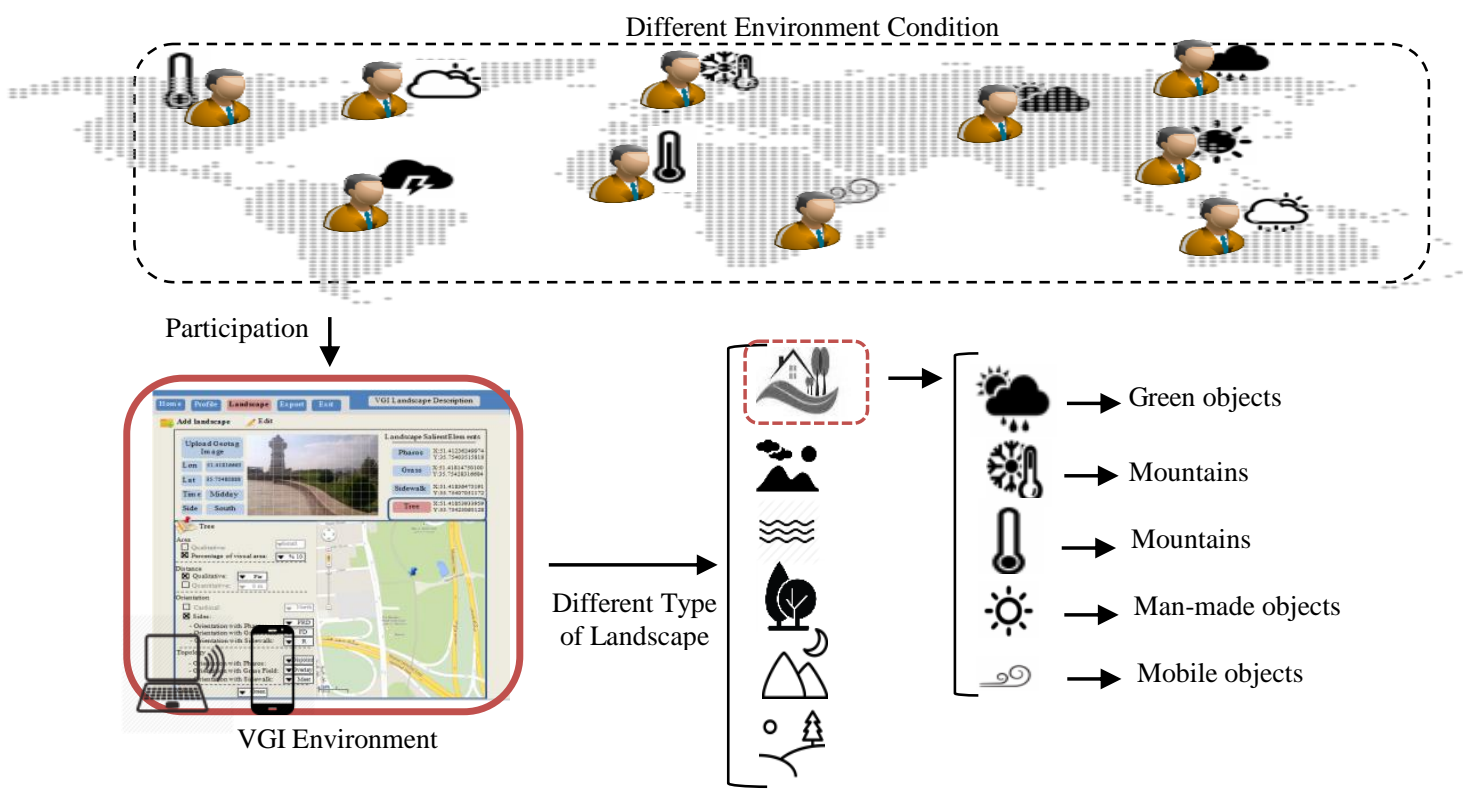

Figure 3. The approach scenario

A.

\section{Defining Metrics}

Some metrics had been defined so as to be used in visual description of landscape. They are, Distance among all elements, Topology relations between elements, Directions by which every element are stand with others, Solid Angle by which every element are seen from where observer is settled, and Salient metric that takes an integral part of one's description. According upon the extravagant human mind's ability, there could be a copious amount of visual measurements in a place; however, the followings summarize the main visual aspects of a landscape that could be described by mentioned metrics.

1) Salience is the most usable metric by which an individual makes a start on describing a landscape.

2) The next metric is Distance that allows users to share information about the configurations of phenomena in a landscape that shows how dense a landscape is.

The Solid Angle is a measure of how large a given object in the landscape will appear to an observer looing to the scenery from a given point of view.

4) As for Direction metric, people use different approaches, such as cardinal points (i.e., north, south, east, or west) and relative orientation relations (i.e., lest, right, back, front, up, and down), so as to recognize directions and landscape configurations.

5) Last but not least, Topological Relations of intersect (i.e., overlap, contains, inside, covers, covered), meet (touch) and disjoint, are used to study and characterize the spatial configurations between regions located in a geographical space.

$B$.

Designing Platform

Then there is needed a participatory environment to make a linkup between people, environment, and managers. Actually, environment, provides a situation for the individuals to create their own account in which they could fill-out some documents about age, disease, preference and taste, to name but a few. This information may be useful to recognize the context of the specific participant.

In this platform individuals can easily attach their geo-tag image and insert as many phenomena in that landscape as they prefer to share with other users.

\section{C.}

\section{Motivation}

Having the psychological information of the participants as well as the spatio-temporal information of various landscapes with different salient phenomena, the system could suggest some Location Based Services in order to encourage users to participate. As a matter of fact, considering both the environmental conditions and the participant's background, we have provided participants with more beneficial wayfinding applications (Soleimani and Malek, 2015).

Moreover, the relation between salient element, environmental condition, and individuals' psychological information including their cognition, preference and even their health condition, allows the web-based VGI environment to offer a location based application that suggests highly appropriate landscape for participant to go there, take a rest and boost their moral (Soleimani, 2015).

D.

\section{Data Mining}

Data mining process leads us to find the relation between every environmental conditions and the chosen element as a salient one. Using web-based platform for gathering landscape description information allows gathering data in a text based file. Such a digital platform eases the way of mining through various and huge amount of information. Precisely, there is embedded in the designed VGI platform a button by which every individual can export the previous information inserted by other participant. 
Considering the purpose of this study, finding the relations between environmental conditions and chosen salient elements in a landscape, we have searched out all the weather condition, light condition, and landscape types, as well as the salient phenomena chosen by individuals in each of those conditions.

E.

\section{Emerging Pattern}

After having obtained specific information from a large spectrum of prepared data, we started to find out the relations between that information. Emerging an accurate pattern of information lead us to notice something valuable about the way environmental condition affects choosing salient object in an area.

\section{INITIAL IMPLEMENTATION AND DISCUSSION}

Knowing the patterns in which the effects of environmental conditions on the selection of salience element could be felt, the information reliability of participatory environment such as VGI environment has improved. Precisely, to validate the proposed system, we performed an experiment on various types of landscape in different environmental conditions during different time of day using 50 suggested images. 50 people including 10 females and 40 males with age ranging from 13 to over 50 are asked to participate. We compared the produced information by participants and the following results have been being obtained:

In the rainy weather, individuals are about to select green objects as a salience one in a landscape. Apart from other sense such as audition, tactile sensation, and sense of smell, by which human beings live their life, the cognition and perceptual ability of human intends them to choose live features that would be more attractive both emotionally and visually in the rainy weather.

In the snowy weather, individuals prefer to select the more elevated objects including mountains as salience object of a landscape. This fact might be related to either the white color of the snow or the accentuated bigness and vastness of the icy mountains.

In sunny weather, the salience object strongly depends on the type of that landscape. In town landscapes, man-made elements is more likely to attract graze, however, in urban landscape participants prefer to choose green objects as salience ones.

In windy weather, the object that is frequently selected as salience one could be every mobile object in that area. Take a flag and a tree branches as an example. However, the color of objects and its color contrast with the context of landscape affects the process of visual selection.

Demonstrated in the result is some of the most important outcome according upon the participants' description. However, considering the fact that the credibility of data of VGI environment revolves around how many users of different psychological information participate to describe landscape in different environmental conditions, this study should be continued.

\section{CONCLUSION}

Whatever the people's cast of mind through choosing a salient element in a landscape is, there are still some environmental conditions that have influence on choosing different objects as the salient element of that area. In fact, as the results showed, these environmental conditions affect the credibility of data produced by people. Furthermore, it is deserved knowing the patterns that selected salient objects have been influenced by. Knowing these patterns, the tourism managers can produce more applicable tourist guide maps thanks to more credible data. However there are too many environmental conditions to be considered to discover how a salient element in a landscape description has been chosen and the proposed platform and approach plays important roles in making a link between human cognition and environmental condition.

\section{REFERENCE}

Cohen, R. (Ed.), 2013. The Development of Spatial Cognition, Psychology Press.

Cornell, E. H., Sorenson, A., \& Mio, T., 2003. Human sense of direction and wayfinding. Annals of the Association of American Geographers, 93(2), 399-425.

Derungs, C., and Purves, R.S., 2013. From Text to Landscape: Locating, Identifying and Mapping the Use of Landscape Features in a Swiss Alpine Corpus, International Journal of Geographical Information Science, 28(6), pp. 1-22.

Flanagin A and Metzger M., 2008. The Credibility of Volunteered Geographic Information, GeoJournal, 72(3), pp. 137-148.

Forati, A., Soleimani, S., Karimipour, F. and Malek, M. R., 2015. Including Users' Semantics in Evaluating the Creditability of Crowedsourced Landscape Descriptions. The International Archives of the Photogrammetry, Remote Sensing and Spatial Information Sciences, Volume XL-3/W3, 2015 ISPRS Geospatial Week 2015, 28 Sep - 03 Oct 2015, La Grande Motte, France.

Litton Jr, R. B., 1968. Forest landscape description and inventories-a basis for landplanning and design.

Schirpke, U., Tasser, E., and Tappeiner, U., 2013. Predicting Scenic Beauty of Mountain Regions. Landscape and Urban Planning, Vol. 111, pp. 1-12.

Siebold, Alisha, and Mieke Donk., 2014. Reinstating salience effects over time: the influence of stimulus changes on visual selection behavior over a sequence of eye movements. Attention, Perception, \& Psychophysics 76.6 (2014): 16551670.

Soleimani, S., Malek, M. R., Claramunt, C., 2015. Qualitative and Quantitative Metrics for the Visual Description of Landscapes in a Volunteered Geographical Information Environment, Submitted.

Soleimani, S. and Malek, M. R., 2015. VGI Improves LBS Wayfinding Services via Emerging Salient Element's Patterns. $12^{\text {th }}$ Symposium on Location Based Services, 16-18 September, LBS 2015 - Technische Universität München.

Soleimani, S., 2015. Landscape Description in Volunteered Geographic Information (VGI) Using Spatial and Temporal Relationships, MSc Thesis, KNT University, Tehran, Iran (In Persian). 
Singer, S., Wang, G., Howard, H., and Anderson, A., 2012. Environmental condition assessment of US Military Installations Using GIS based spatial multi-criteria decision analysis. Environmental management, 50(2), 329-340.

Williams, Maia, Werner Kuhn, and Marco Painho., (2015)The influence of landscape variation on landform categorization." Journal of Spatial Information Science 5: 5173.

Yaouanc, L., Saux, J.M. and Claramunt, C., 2010. A Semantic and Language-based Representation of an Environmental Scene. Geoinformatica, 14(3), pp. 333-352.

Yildirim, Gökhan, and Sabine Süsstrunk., 2015. FASA: fast, accurate, and size-aware salient object detection. Computer Vision--ACCV 2014. Springer International Publishing. 514528.

Zhang, Duzhen, and Chuancai Liu., 2014. A salient object detection framework beyond top-down and bottom-up mechanism. Biologically Inspired Cognitive Architectures 9 2014: 1-8. 